\title{
Asteroid masses and improvement with Gaia
}

\author{
S. Mouret ${ }^{1}$, D. Hestroffer ${ }^{1}$, and F. Mignard ${ }^{2}$ \\ 1 IMCCE, UMR CNRS 8028, Paris observatory, 77 Av. Denfert-Rochereau, 75014 Paris, France \\ e-mail: [mouret; hestroffer] @imcce.fr \\ 2 OCA/Cassiopée, UMR CNRS 6062, Nice observatory, Le Mont Gros, BP 4229, 06304 Nice Cedex 4, France \\ e-mail: francois.mignard@obs-nice.fr
}

Received 14 March 2007 / Accepted 18 May 2007

\section{ABSTRACT}

\begin{abstract}
Context. The ESA astrometric mission Gaia, due for launch in late 2011, will observe a very large number of asteroids ( 350000 brighter than $V=20$ ) with an unprecedented positional precision (at the sub-milliarcsecond level).

Aims. Gaia will yield masses of the hundred largest asteroids from gravitational perturbations during close approaches with target asteroids. The objective is to develop a global method to obtain these masses which will use simultaneously all perturbers together with their target asteroids. In this paper, we outline the numerical and statistical methodology and show how to estimate the accuracy. Methods. The method that we use is the variance analysis from the formulation of observed minus calculated position $(\mathrm{O}-\mathrm{C})$. We assume a linear relationship between the $(\mathrm{O}-\mathrm{C})$ and the fitted parameters which are the initial position and velocity of the asteroids and the masses of perturbers. The matrix giving the partial derivatives with respect to the positions and masses is computed by numerical integration of the variational equations.

Results. We show that with Gaia it will be possible to derive more than 100 masses of minor planets 42 of which with a precision better than $10 \%$.
\end{abstract}

Key words. astrometry - celestial mechanics

\section{Introduction}

Gaia is an astrometric cornerstone mission of the European Space Agency. With a launch due in late 2011, Gaia will have a much more ambitious program than its precursor Hipparcos: to create a "3D map" of our galaxy from astrometric, photometric and spectroscopic observations (Mignard 2005). It will pinpoint one billion targets with an unprecedented positional precision (at the sub-milliarcsecond level for single observation) and will also observe about 350000 asteroids (mainly main belt asteroids) brighter than $V=20$.

From the positions of a subset of asteroids selected according to certain criteria detailed later in this paper, the Gaia data processing should yield the masses of the $\approx 100-200$ most massive asteroids of the main belt. These precise determinations will be of utmost significance for the improvement of the dynamical modelling of the solar system and also for our knowledge of the physics of asteroids. As usual the method used for deriving the masses is based on the analysis of orbital perturbations during close encounters between the few massive asteroids (hereafter referred to as the perturbers) and numerous smaller asteroids (the targets). We show in this paper that the number of significant close approaches involving many perturbers imposes global processing of the whole set of perturbers and the target asteroids in a single fit that takes the masses as global parameters. This is a real challenge due to the size of the data set and the coupling between the positional and physical parameters whose solution requires significant preparation on simulated data. Although this is developed in the framework of the Gaia data processing, the method is quite general and applicable to ground-based data for which it should improve the statistical significance of the results.

From a dynamical point of view, the knowledge of the asteroid masses will allow precise long-term orbits of several smaller objects that are perturbed by larger asteroids to be obtained. More importantly, it will enable an increase in the accuracy of the solar system ephemeris, knowing that currently the limiting factor is not the dynamical modelling or the observation fitting, but the lack of reliable masses of the largest asteroids and more globally that the main belt perturbs the motion of the planet like a ring of matter. This uncertainty is particularly relevant for Mars (Standish et al. 2002).

From a physical point of view, the mass and size (from IRAS but also from Gaia) of an asteroid yields its bulk density, which accounts for the amount of matter making up the body and the space occupied by its pores and fractures. The calculation of the porosity, giving an idea of the part of the void which makes up the asteroid, is of great interest to understand the formation of these small bodies. It directly depends on the ratio between the bulk density of the asteroid and the sample density of its material, where the latter can be estimated from the grain density of a meteorite possessing a similar composition. Knowing that during a collision, the internal structure of an asteroid acts upon the stress-wave propagation, which in turn, modifies its constitution, the porosity becomes a precious indicator of the collisional history of asteroids and of their internal structure (Britt et al. 2002).

In addition, a measure of the bulk density will shed light on the relationship between the spectroscopic taxonomic class (also available from Gaia) and the density, or possibly the porosity. Such progress could provide information on their origins and on the formation process of the solar system. If a well-defined relationship can be shown, we could make reliable density estimates for objects of known taxonomic classes.

Similarly, the comparison between these densities for objects belonging to different taxonomic classes and the density of meteorites may strengthen our ideas about the genetic relationship 
existing between different classes of meteorites and their supposed parent asteroids (Zappalà et al. 2002).

In Sect. 2 we focus on the relevant specificities of the observations of the solar system objects by Gaia; then in Sect. 3 we explain how the target asteroids are selected from the mutual close approaches. Section 4 is the heart of the paper with the description and validation of the global mathematical problem raised by the repeated Gaia observations. The expected final accuracy on the masses based on an extensive simulation is given and analysed in the last section.

\section{Ground and space-based observations}

\subsection{A quick survey of the known asteroid masses}

The first mass determination of an asteroid only goes back to 1966, although the principles of assessing masses of major planets or satellites from gravitational perturbations had been applied for more than a century. Hertz (1966) estimated the mass of 4 Vesta from the analysis of its gravitational pull on a smaller target asteroid, 197 Arete. This method was applied successfully for the first time to an asteroid and has remained the only usable method if we exclude the very specific cases of space probes flyby. Hilton (2002) reported 24 asteroids whose mass has been determined by considering the perturbation of a test asteroid, a planet (Mars) or a spacecraft, as well as the observation of the companion for binary asteroids. Twelve recently determined new masses by the classical dynamical method (see Table 1, in bold), must be added today to the Hilton list: nine were estimated by Kochetova (2004) and three others by Kuznetsov (2001), Chesley (2005) and Aslan et al. (2006). In addition, six more precise masses have been derived for the asteroids: 15 Eunomia by Vitagliano (2006), 52 Europa, 444 Gyptis and 511 Davida by Kochetova (2004), 16 Psyche by Kuzmanoski et al. (2002) and 433 Eros by Miller et al. (2002). The observation of a satellite for binary asteroids in the main belt also brought 8 new masses with 4 estimated by Behrend et al. (2006), three by Marchis et al. $(2003,2006)$ and one by Descamps et al. (personal communication).

The distribution of the relative precisions on the masses in Table 1 shows that our knowledge of the asteroid masses is still very poor. Even if the masses of the largest asteroids are derived with relatively good precision, sometimes reaching the 0.2 percent level (Ceres), the uncertainty in the value of masses from estimates given by Hilton (2002) are generally between 10 and $20 \%$. The scatter in the estimates of the mass of a given body derived during several independent encounters is a first hint at the external accuracy of the determination, and not surprisingly this scatter is larger than the typical dispersion expected from the formal accuracies. As an illustration, we considered 17 independent results for the mass of Ceres (Table 2) among them, the 13 mass determinations and their associated precision referenced in Hilton's paper (2002), as well as 4 other more recent determinations from Kovačević \& Kuzmanoski (2005).

In Fig. 1, we have plotted these different mass estimations $m_{i}$ with their error bars $\sigma_{i}$. The weighted mean is shown with the horizontal dashed line at $4.769 \times 10^{-10} M_{\odot}$. The histogram gives for each mass its reduced value $\left|\left(m_{i}-\mu\right)\right| / \sigma_{i}$ so that the determinations in strong disagreement with the weighted mean are easily spotted. Three values ( $\sim 18 \%$ of the total) appear to be outliers above the $3 \sigma$ level.
The evaluation of the standard deviation of the weighted mean $\mu_{w}$, by using the expression,

$\bar{\sigma}_{\mu_{w}}=\frac{1}{\sqrt{\sum_{i=1}^{n} w_{i}}}$ with $w_{i}=\frac{1}{\sigma_{i}^{2}}$

gives us a good enough deviation of,

$\bar{\sigma}_{\mu_{w}}=0.0077 \times 10^{-10} M_{\odot}$

However, this standard deviation is underestimated because the errors $\sigma_{i}$ are formal error and cannot account for systematic errors which become relevant when comparing a set of measurements. The systematic errors may have many origins like the incompleteness of the dynamical model, whose effect is shown by Michalak (2000). This point is illustrated by deriving the masses of three massive asteroids with or without including perturbing asteroids in its dynamical model. The results show that the formal errors of each determination remain quite similar in both cases while the masses themselves change with the model.

In some cases, the correlation which can exist between the a priori mass of certain target asteroids and the perturber can cause further error on the computed mass. Hilton (2002) cites the example of Pallas and states that this correlation is due to the similarity in the mean motions and mean longitude in the case of Ceres perturbing Pallas. Goffin's analysis (2001) puts forward other causes: the high inclination and eccentricity of Pallas is the origin of the small number of significant close approaches for the mass determination.

In addition to these sources of errors which do not allow estimation of the true deviation associated with each measure $m_{i}$, the standard deviation $\bar{\sigma}_{\mu_{w}}$ of the weighted mean is well-known as underestimating the variance (Zhang 2006). Nevertheless, even if we can estimate the standard deviation by a more realistic formula such as Eq. (2) employed by Michalak (2000), it does not take into account the bias of each method.

$\sigma_{\mu_{w}}=\sqrt{\frac{s^{2}}{\sum_{i=1}^{n} w_{i}}}$

where,

$s^{2}=\frac{1}{n-1} \sum_{i=1}^{n} w_{i}\left(m_{i}-\mu_{w}\right)^{2}$.

Thus, Viateau \& Rapaport (1995) for the mass of Ceres, increased the standard deviation $\sigma=0.1 \times 10^{-10}$ by a factor 2 to compensate for the underestimation of the evaluated uncertainty. However the problem remains and it is in practice very difficult to estimate the value of the uncertainty in the derivation of the masses of the asteroids by gravitational means.

\subsection{Observations with Gaia}

Gaia will observe the sky from its platform located in the vicinity of the Sun-Earth Lagrange point L2, approximately 1.5 million $\mathrm{km}$ away from the Earth in the Sun-Earth direction. This region will provide a stable radiation and thermal environment to the spacecraft with a relatively easy link to the ground stations. Around the L2 point, Gaia will follow a large Lissajous orbit $(\sim 300000 \mathrm{~km})$ to avoid the eclipse of the Sun by the Earth so as to maintain a constant power supply and thermal flux. During the operations, the two astrometric fields of Gaia (separated by an angle of 106.5 degrees) will scan the sky at a constant rate of $60 \operatorname{arcsec~s}^{-1}$. As a consequence, over a period of $6 \mathrm{~h}$ (the spin 
Table 1. The best precisions of asteroid mass determinations. The new asteroids for which the mass has been determined using the dynamical method since Hilton's paper (2002) appear in bold.

\begin{tabular}{|c|c|c|c|c|c|}
\hline \multicolumn{2}{|c|}{$\begin{array}{l}\text { Asteroid } \\
n \text { name }\end{array}$} & \multirow{2}{*}{$\begin{array}{c}\text { Mass } \\
{\left[M_{\odot}\right]} \\
(4.81 \pm 0.01) \times 10^{-10}\end{array}$} & \multirow{2}{*}{$\begin{array}{c}\text { Relative precision } \\
\sigma(m) / m \\
0.2 \%\end{array}$} & \multirow{2}{*}{$\begin{array}{l}\begin{array}{c}\text { Mass determination } \\
\text { method }\end{array} \\
\text { Mars pertubation }\end{array}$} & \multirow{2}{*}{$\begin{array}{l}\text { Reference } \\
\text { Pitjeva (2001) }\end{array}$} \\
\hline 1 & Ceres & & & & \\
\hline $2 \mathrm{I}$ & Pallas & $(1.00 \pm 0.01) \times 10^{-10}$ & $1.0 \%$ & Mars pertubation & Pitjeva (2001) \\
\hline 3 & Juno & $(2.09 \pm 0.35) \times 10^{-11}$ & $16.7 \%$ & asteroid pertubation & Kochetova (2004) \\
\hline 4 & Vesta & $(1.36 \pm 0.01) \times 10^{-10}$ & $0.7 \%$ & Mars pertubation & Pitjeva (2001) \\
\hline 6 & Hebe & $(0.69 \pm 0.09) \times 10^{-11}$ & $13.0 \%$ & asteroid perturbation & Kochetova (2004) \\
\hline 7 & Iris & $(1.41 \pm 0.14) \times 10^{-11}$ & $9.9 \%$ & asteroid perturbation & Kochetova (2004) \\
\hline 10 & Hygiea & $(4.54 \pm 0.13) \times 10^{-11}$ & $2.9 \%$ & asteroid perturbation & Chesley (2005) \\
\hline 11 & Parthenope & $(2.56 \pm 0.07) \times 10^{-12}$ & $2.7 \%$ & asteroid perturbation & Viateau \& Rapaport (2001) \\
\hline 15 & Eunomia & $(1.64 \pm 0.06) \times 10^{-11}$ & $3.7 \%$ & asteroid perturbation & Vitagliano (2006) \\
\hline \multirow[t]{2}{*}{16} & Psyche & $(1.34 \pm 0.22) \times 10^{-11}$ & $16.4 \%$ & asteroid perturbation & Kochetova (2004) \\
\hline & & $(3.38 \pm 0.28) \times 10^{-11}$ & $8.3 \%$ & asteroid perturbation & Kuzmanoski et al. (2002) \\
\hline 20 & Massalia & $(2.44 \pm 0.40) \times 10^{-12}$ & $16.4 \%$ & asteroid perturbation & Bange (1998) \\
\hline \multirow[t]{2}{*}{22} & Kalliope & $(0.85 \pm 0.28) \times 10^{-11}$ & $32.9 \%$ & asteroid perturbation & Kochetova (2004) \\
\hline & & $(0.40 \pm 0.013) \times 10^{-11}$ & $3.1 \%$ & observation of satellite & Marchis et al. (2003) \\
\hline 29 & Amphitrite & $(0.77 \pm 0.13) \times 10^{-11}$ & $16.9 \%$ & asteroid perturbation & Kochetova (2004) \\
\hline 31 & Euphrosyne & $(0.94 \pm 0.52) \times 10^{-11}$ & $55.3 \%$ & asteroid perturbation & Kochetova (2004) \\
\hline \multirow[t]{2}{*}{45} & Eugenia & $(3.0 \pm 0.1) \times 10^{-12}$ & $3.3 \%$ & observation of satellite & Merline et al. (1999) \\
\hline & & $(2.82 \pm 0.03) \times 10^{-12}$ & $1.1 \%$ & observation of satellite & Descamps et al. ${ }^{4}$ \\
\hline 48 & Doris & $(0.61 \pm 0.30) \times 10^{-11}$ & $49.1 \%$ & asteroid perturbation & Kochetova (2004) \\
\hline 52 & Europa & $(1.27 \pm 0.25) \times 10^{-11}$ & $19.6 \%$ & asteroid perturbation & Kochetova (2004) \\
\hline 65 & Cybele & $(0.58 \pm 0.15) \times 10^{-11}$ & $25.9 \%$ & asteroid perturbation & Kochetova (2004) \\
\hline 87 & Sylvia & $(7.39 \pm 0.03) \times 10^{-12}$ & $0.4 \%$ & observation of satellite & Marchis (2005) \\
\hline 88 & Thysbe & $(7 \pm 1) \times 10^{-12}$ & $14.2 \%$ & asteroid perturbation & Michalak (2001) \\
\hline \multirow[t]{2}{*}{90} & Antiope & $(4.14 \pm 0.05) \times 10^{-13}$ & $1.2 \%$ & observation of satellite & Merline et al. (2002) \\
\hline & & $(4.05 \pm 0.24) \times 10^{-13}$ & $6.0 \%$ & observation of satellite & Descamps et al..$^{5}$ \\
\hline 121 & Hermione & $(2.7 \pm 0.015) \times 10^{-12}$ & $0.6 \%$ & observation of satellite & Marchis et al. (2005) \\
\hline 165 & Loreley & $(1.57 \pm 1.0) \times 10^{-11}$ & $63.7 \%$ & asteroid perturbation & Aslan et al. (2006) \\
\hline \multirow[t]{2}{*}{243} & Ida & $(2.2 \pm 0.3) \times 10^{-14}$ & $13.6 \%$ & observation of satellite ${ }^{1}$ & Petit et al. (1997) \\
\hline & & $(2.1 \pm 0.3) \times 10^{-14}$ & $14.3 \%$ & asteroid perturbation & Belton et al. (1995) \\
\hline 253 & Mathilde & $(5.19 \pm 0.02) \times 10^{-14}$ & $0.4 \%$ & spacecraft perturbation & Yeomans et al. (1998) \\
\hline 324 & Bamberga & $(2.29 \pm 0.38) \times 10^{-11}$ & $16.6 \%$ & asteroid perturbation & Kochetova (2004) \\
\hline 379 & Huenna & $(2.38 \pm 0.25) \times 10^{-13}$ & $10.5 \%$ & observation of satellite & Marchis et al. $(2005)^{2}$ \\
\hline 433 & Eros & $(3.345 \pm 0.0015) \times 10^{-15}$ & $0.05 \%$ & spacecraft perturbation & Miller et al. (2002) \\
\hline 444 & Gyptis & $(0.63 \pm 0.12) \times 10^{-11}$ & $19.0 \%$ & asteroid perturbation & Kochetova (2004) \\
\hline 451 & Patientia & $(3.21 \pm 0.85) \times 10^{-11}$ & $22.4 \%$ & asteroid perturbation & Kuznetsov (2001) \\
\hline 511 & Davida & $(2.40 \pm 0.24) \times 10^{-11}$ & $10.0 \%$ & asteroid perturbation & Kochetova (2004) \\
\hline 532 & Herculina & $(1.68 \pm 0.28) \times 10^{-11}$ & $16.6 \%$ & asteroid perturbation & Kochetova (2004) \\
\hline 617 & Patroclus & $(0.58 \pm 0.05) \times 10^{-12}$ & $8.7 \%$ & observation of satellite & Marchis et al. (2006) \\
\hline 624 & Hektor & $(4.97 \pm 0.06) \times 10^{-12}$ & $1.2 \%$ & observation of satellite & Descamps et al. ${ }^{4}$ \\
\hline 704 & Interamnia & $(3.5 \pm 0.9) \times 10^{-11}$ & $25.7 \%$ & asteroid perturbation & Michalak (2001) \\
\hline 762 & Pulcova & $(1.28 \pm 0.02) \times 10^{-12}$ & $1.6 \%$ & observation of satellite & Merline et al. (2002) \\
\hline 854 & Frostia & $(1.06 \pm 0.95) \times 10^{-15}$ & $89.6 \%$ & observation of satellite ${ }^{3}$ & Behrend et al. (2006) \\
\hline 1089 & 9 Tama & $(8.9 \pm 3.2) \times 10^{-16}$ & $35.9 \%$ & observationof satellite ${ }^{3}$ & Behrend et al. (2006) \\
\hline 1313 & 3 Berna & $(2.25 \pm 2.01) \times 10^{-15}$ & $89.3 \%$ & observation of satellite ${ }^{3}$ & Behrend et al. (2006) \\
\hline 4492 & 2 Debussy & $(2.97 \pm 2.6) \times 10^{-16}$ & $87.5 \%$ & observation of satellite $^{3}$ & Behrend et al. (2006) \\
\hline
\end{tabular}

${ }^{1}$ The mass of 243 Ida was estimated by numerical simulations considering that its orbit is stable, and not from observations.

2 URL: http://astro.berkeley.edu/ fmarchis/Science/Asteroids/Huenna.html

${ }^{3}$ The authors only gave an interval for the mass value. We considered a Gaussian distribution to compute the standard deviation of the mass determination.

${ }^{4}$ Personal communication.

${ }^{5}$ Icarus, in press.

period) the two fields of view will scan across all objects located along a great circle perpendicular to the instantaneous spin axis. This spin axis will precess with a period of 70 days around the solar direction keeping a fixed angle of 45 degrees with the solar direction. As a consequence, the sky will be entirely covered in 6 months and each object will be observed about 70 times on average during the mission lifetime of five years.
The position of objects observed by Gaia will be expressed in longitude $\lambda$ and latitude $\beta$ on a reference great circle (RGC) (see Fig. 3), computed from the mean positions of instantaneous scanning circles (their rotation pole $z$ being never fixed) over one period. These observations are used to describe the spacecraft attitude during the mission. Along-scan measurements (and the RGC longitude) are much more accurate than those in the 
Table 2. Different mass determinations of Ceres computed between 1991 and 2005.

\begin{tabular}{cccl}
\hline \hline $\begin{array}{c}\text { Index } \\
i\end{array}$ & $\begin{array}{c}\text { Mass } m_{i} \\
{\left[10^{-10} M_{\odot}\right]}\end{array}$ & $\begin{array}{c}\text { error } \sigma_{i} \\
{\left[10^{-10} M_{\odot}\right]}\end{array}$ & Reference \\
\hline 1 & 4.7 & 0.3 & Goffin (1991) \\
2 & 4.796 & 0.085 & Sitarski et al. (1992) \\
3 & 4.8 & 0.22 & Williams (1992) \\
4 & 4.622 & 0.071 & Sitarski et al. (1995) \\
5 & 5.0 & 0.2 & Viateau et al. (1995) \\
6 & 4.71 & 0.09 & Carpino et al. (1996) \\
7 & 4.26 & 0.09 & Kuzmanoski (1996) \\
8 & 4.785 & 0.039 & Viateau et al. (1997) \\
9 & 4.759 & 0.023 & Viateau et al. (1998) \\
10 & 4.39 & 0.04 & Hilton (1999) \\
11 & 4.7 & 0.04 & Michalak (2000) \\
12 & 4.76 & 0.02 & E. M. Standish ${ }^{1}$ \\
13 & 4.81 & 0.01 & Pitjeva (2001) \\
14 & 4.63 & 0.26 & Kovačević et al. (2005) \\
15 & 4.6 & 0.29 & Kovačević et al. (2005) \\
16 & 5.02 & 0.44 & Kovačević et al. (2005) \\
17 & 5.05 & 0.76 & Kovačević et al. (2005)
\end{tabular}

${ }^{1}$ Personal communication (2001).
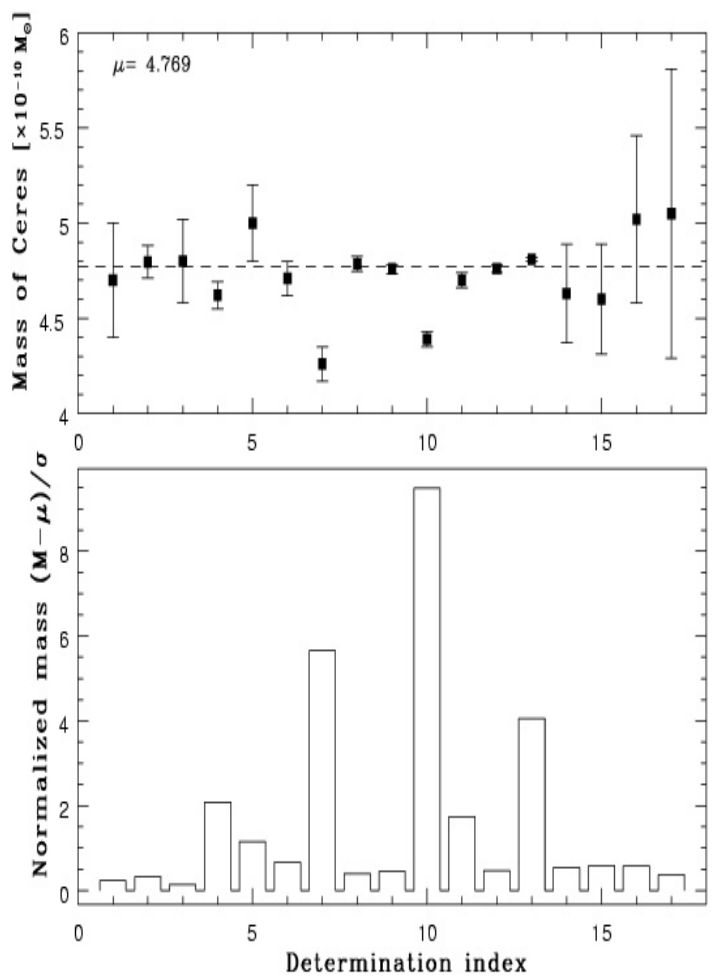

Fig. 1. Top: different mass determinations of 1 Ceres. The masses are in $10^{-10} M_{\odot}$ unit. Bottom: distribution of the different measures $m_{i}$ of the mass of 1 Ceres according to the normalise variable $\left(m_{i}-\mu\right) / \sigma_{i}$.

transverse direction (i.e. RGC latitude) and thus in the following we have only considered the astrometric information coming from the Gaia RGC longitudes.

As is the case for observations of minor planets, Figs. 4-5 illustrate with the asteroid 1000 Piazzia the time distribution of observations and the orbit coverage that Gaia will permit for most of the bodies of the main-belt. The mission lifetime of five years matches fairly well with the typical orbital period of the main-belt asteroids and the regular return of the pointing to a

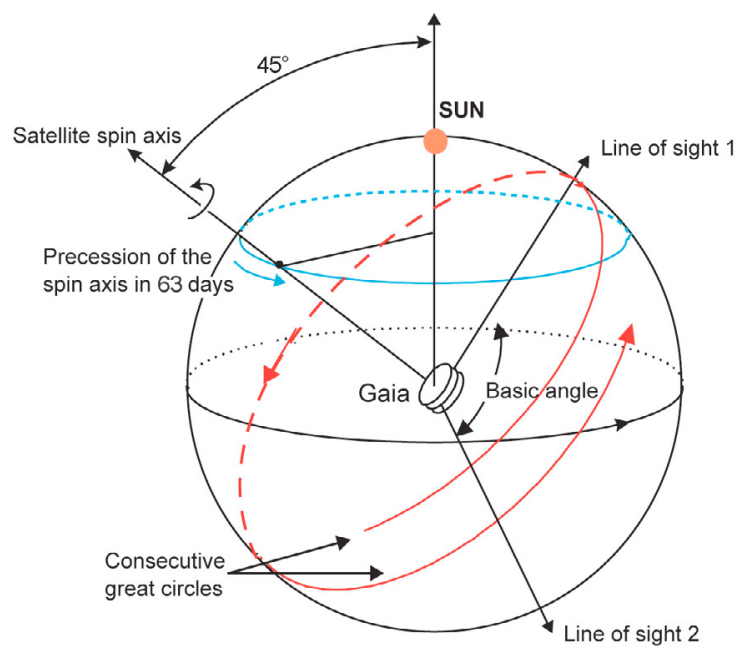

Fig. 2. Gaia's two fields-of-view scan the sky according to a carefully prescribed scanning law. The constant spin rate of $60 \mathrm{arcsec} \mathrm{s}^{-1}$ corresponds to 6-h great-circle scans. The angle between the slowly precessing spin axis and the Sun is maintained at $45^{\circ}$ and the basic angle is $106.5^{\circ}$.

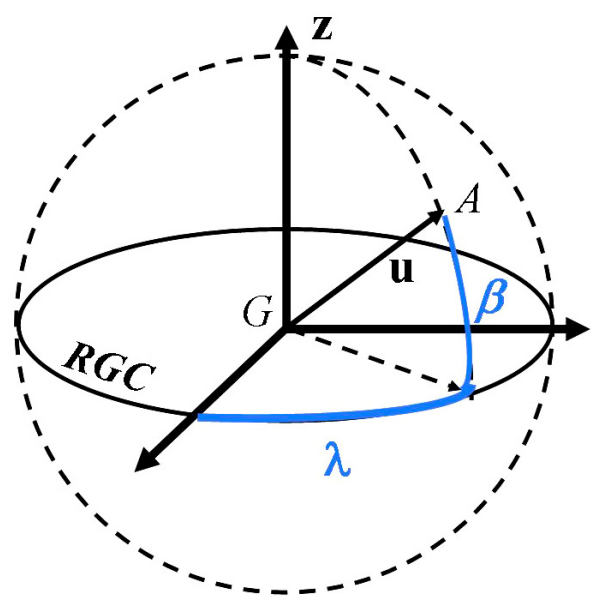

Fig. 3. The Gaia longitude $\lambda$ and latitude $\beta$ of an object position projected on the RGC. The frame is centered on the Gaia satellite G. The vector $\boldsymbol{z}$ is the satellite spin axis and $\boldsymbol{u}$ is the direction vector toward the observed asteroid $A$ from the Gaia spacecraft $G$.

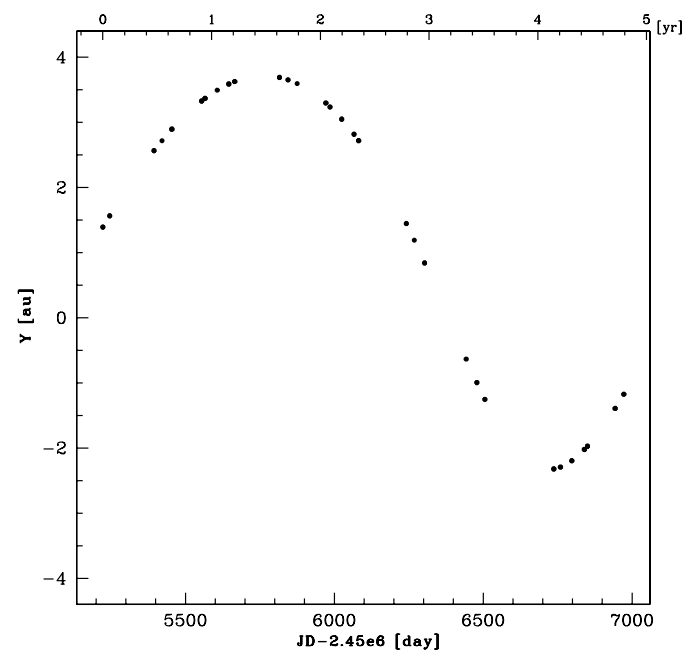

Fig. 4. Time distribution of Gaia observations for one arbitrary typical main-belt asteroid (1000 Piazzia). 


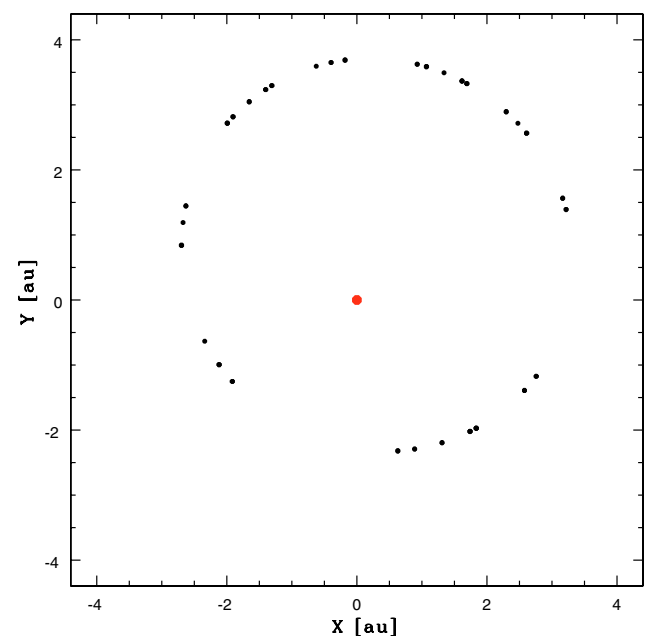

Fig. 5. Spatial distribution of Gaia observations for one arbitrary typical main-belt asteroid (1000 Piazzia).

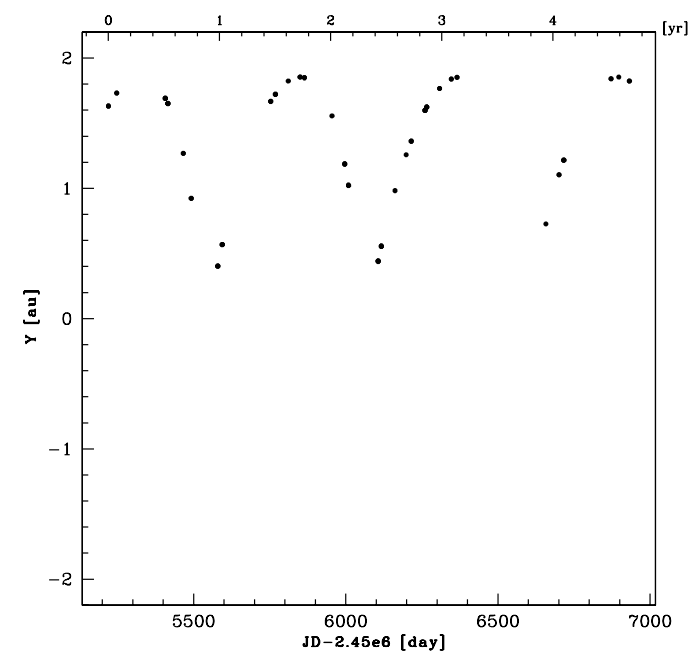

Fig. 6. Time distribution of Gaia observations for one arbitrary nearearth asteroid (3200 Phaeton).

target during this period. These are both very favorable circumstances for solar system science with Gaia. Close approaches will be more or less regularly distributed during the mission allowing identification of the difference in osculating elements in the arcs before and after the close approach. In practice a single orbit per body will be fitted to the observations adding the mass of the perturbers as additional unknowns.

Concerning the Near-Earth asteroids, several will be well observed over their orbit as shown with the example of 3200 Phaeton in Figs. 6-7. Given the limiting magnitude of Gaia at $V \approx 20$ and the estimated level of completeness of current surveys, the number of detectable objects with the spacecraft should be 350000 asteroids from the main belt. A few additional Trojans and TNOs will also be observed together with about 1000 NEOs, but for the latter population there will be a fairly irregular coverage due to their large apparent magnitude excursion (Fig. 8).

Starting from the orbits and absolute magnitudes of known objects given by the ASTORB database available online, one can compute the average distances to the Earth and to the Sun

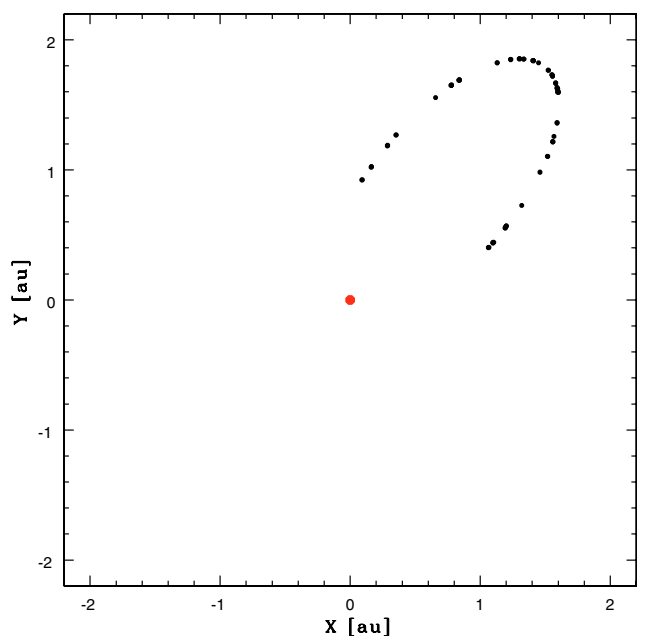

Fig. 7. Spatial distribution of Gaia observations for one arbitrary nearearth asteroid (3200 Phaeton).

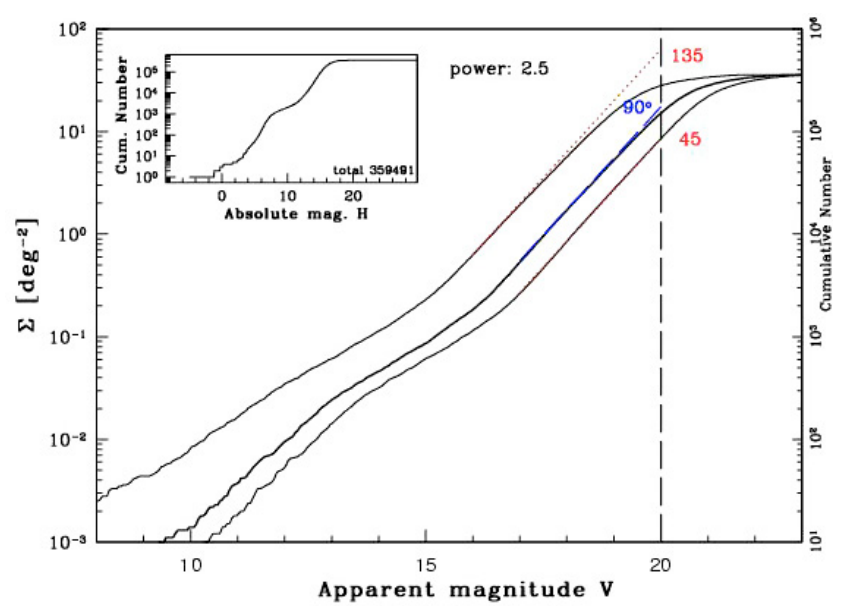

Fig. 8. Cumulative distribution in apparent $V$ magnitude for all known objects with $q>1$. The inset gives the distribution in absolute magnitude. The surface density $\Sigma$ on the left label is a mean based on an ecliptic band of \pm 30 degrees. Average apparent magnitudes are computed for 3 different solar elongations.

for various solar elongations and estimate the apparent magnitude as:

$V=H+5 \log (r \cdot \Delta)+f(\alpha)$

where $r$ and $\Delta$ are respectively the heliocentric and geocentric distances and $f(\alpha)$ is the phase photometric factor. An analysis of the yearly evolution of the database content as a function of the absolute magnitude has shown that one can consider that completeness is achieved at absolute magnitude $H=14$ and close to $80 \%$ at $H=15$. Extrapolation of the curves considering a power low of index 2.5 gives the predicted number of objects for each solar elongation observable by Gaia. Were all objects observed regularly at 135 degrees of elongation, Gaia would have been able to observe about 600000 planets. The actual geometry is not so favorable and the current guess is that Gaia will have a good time sampling on about 350000 asteroids.

The single observation precision is plotted in Fig. 9 as a function of the apparent magnitude during the transit of the field of view. Each point results from a simulation of the detection of an asteroid taking into account the CCD properties and the transit velocity. The data can be fitted to a simple expression used later 


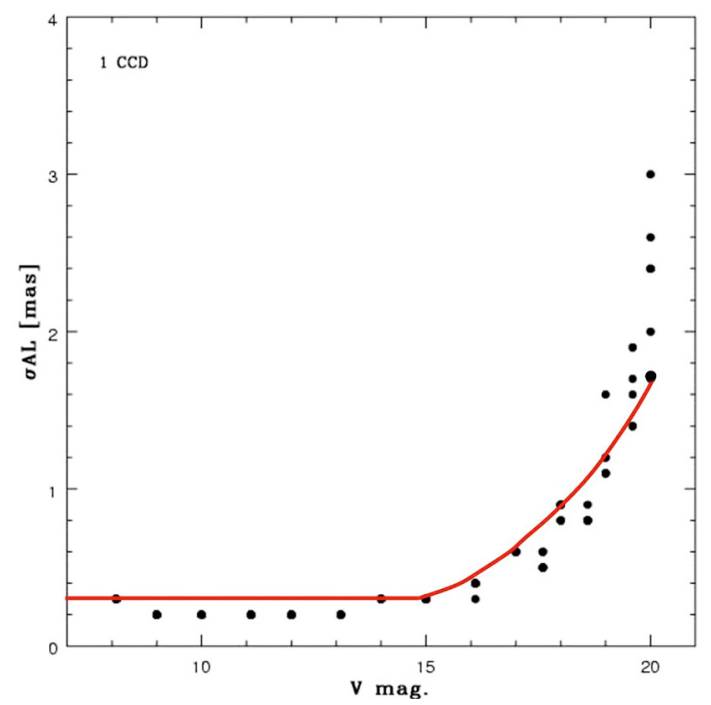

Fig. 9. Effect of the apparent magnitude on the single observation precision $\sigma$ of the Gaia longitude $\lambda$ of an asteroid.

in this paper to model the observation accuracy as,

$\sigma=310 \mu$ as for $V \leq 15$

$\sigma=10^{0.147 V-2.71}$ mas for $V>15$.

A single observation here means a single transit with the combination into a single measurement of the 9 CCDs records performed during this transit.

With 350000 possible targets in the main belt over five years the number of efficient close approaches will be very large, at least for the largest bodies. Therefore their mass will result from the perturbation of several tens or even hundreds of small bodies, a circumstance that will seriously limit the risk of systematic effects while improving the statistical significance of the mass.

\section{The selection of target asteroids}

An important step for mass determination is the selection of suitable target over which the gravitational pull of a larger asteroid is detectable. The classical criteria to select the interesting encounters are (i) the minimum distance between the target asteroid and the perturber and (ii) the deflection angle $\theta$ of the path of the perturbed asteroid. This angle gives in many cases a good approximation of the magnitude of the perturbation to which the target asteroid is subject. It is determined according to a twobody ballistic approach where the relative trajectory of the target with respect to the perturber can be modelled by a hyperbola (see Fig. 10).

From the hyperbolic two-body problem the deflection angle $\theta$ is given by,

$\tan \frac{\theta}{2}=\frac{G(m+M)}{v^{2} b}$

where $G$ is the constant of gravitation, $M$ is the mass of perturber, $m$ that of the target asteroid, $v$ the relative velocity of the encounter and $b$ the impact parameter which can be reduced to the minimal distance between the two asteroid trajectories in the case where we do not take into account their mutual perturbations.

In the following simulations the target asteroids are selected among the first 20000 numbered asteroids using a software developed for the Gaia mission by a member of our group (FM)

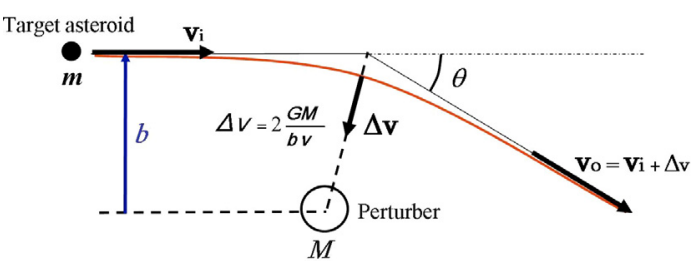

Fig. 10. Impulse approximation of a small target asteroid perturbed by a larger one. $v_{\mathrm{i}}$ and $v_{\mathrm{o}}$ are respectively the incoming and outgoing velocity vector. The effect of the perturbation is expressed by the vector $\Delta v$.

which searches systematically all the close approaches within a set of bodies over a prescribed duration. The perturbers are also chosen among the 20000 asteroids as the bodies having a mass larger than a given threshold (here set at $10^{-13} M_{\odot}$ ). The program starts from a list of orbital elements at a given epoch and determines the positions of the asteroids with a two-body problem between two epochs. It then computes for each pair of selected asteroids the minimum distance by an iterative vector parabolic fit. In practice, this software proves to be very fast and precise enough to locate the close approach for a period not exceeding 10 years in the case where a single set of osculating elements is used over that period. Once a close approach has met the criterion on the minimal distance, the angular deflection is evaluated by Eq. (3) and a second filter is applied by imposing a threshold value below which the close approach is not taken into account for the mass determination.

The program was run from 2010.5 to 2016, corresponding approximately to the expected observing period of Gaia. Table 3 provides the number of close approaches for a set of criteria, the number of perturbers and target asteroids that were found, as well as the number of bodies which are both perturber and targets.

The number of significant close approaches (significant in relation to the Gaia astrometric accuracy) is huge in comparison with the few isolated cases available with ground-based observations and sub-arcsec astrometry. These approaches are fairly uniformly distributed over the Gaia lifetime with a small scatter over the years as shown in Fig. 11 on the histogram with 2-month bins. In this plot, a close approach has been selected when the minimum distance between the two bodies is less than $0.5 \mathrm{AU}$ and the deflection angle computed with the impulse formula is $>1$ mas. A close approach occurring close to the start or the end of the observation period will not carry much information on the perturber mass, since the orbit of the target (assuming no other close approach during the Gaia mission) would be unperturbed during the observation interval. Dedicated ground based observations before or after the mission could benefit this close approach by combining the two sets of observations. This would be valuable for the strong perturbations only, given the accuracy of the ground-based observations compared to Gaia.

\section{The method for mass determination}

\subsection{The $\mathrm{O}-\mathrm{C}$ linearized formulation}

The method to derive the masses is based on the fitting of the initial conditions of target asteroids to the observed positions, by including in the dynamical model the masses and the orbits of the perturbers. One starts from reasonable values for the initial conditions and the masses of the perturbers and the goal is to compute the corrections to the initial conditions of the asteroid orbit by minimizing the differences between the observed and computed positions $(\mathrm{O}-\mathrm{C})$. The observations are expressed in 
Number of close approaches

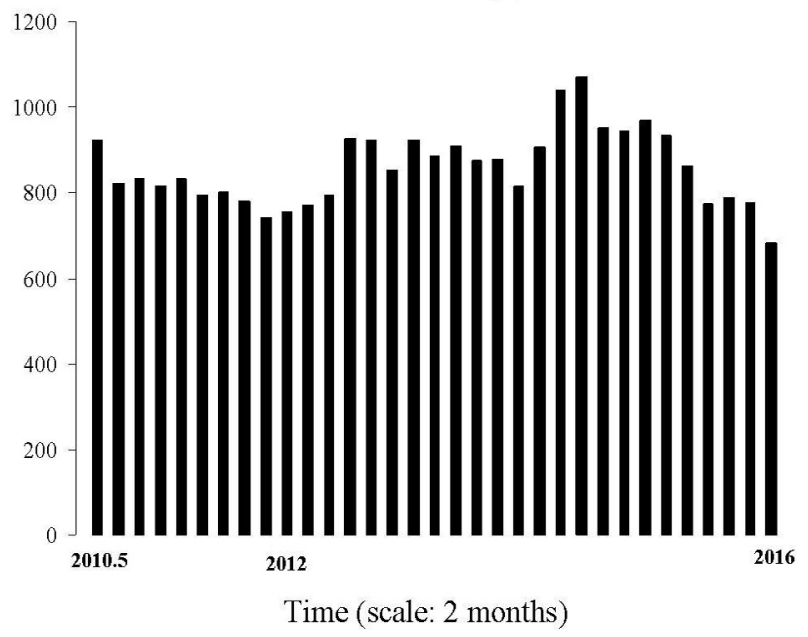

Fig. 11. Distribution of the number of close approaches every 2 months from 2010.5 to 2016 with a minimum distance $<0.5 \mathrm{AU}$ and deflexion angle $>1$ mas.

Gaia longitude $\lambda$ projected over a given Great Circle. Thus we express the vector $(\mathrm{O}-\mathrm{C})$ by a linear relation (4) as a function of the free parameters, namely the corrections to the initial position and velocity of each target and the masses (or their difference to a reference) of the perturbers $\left(\delta \boldsymbol{u}^{0}, \delta \boldsymbol{m}_{\mathrm{p}}\right)^{t}$. The overdetermined system is solved by least squares as

$\mathrm{O}-\mathrm{C}=\mathbf{A}\left(\begin{array}{c}\delta \boldsymbol{u}_{0} \\ \delta \boldsymbol{m}_{\mathrm{p}}\end{array}\right) \Rightarrow\left(\begin{array}{c}\delta \boldsymbol{u}_{0} \\ \delta \boldsymbol{m}_{\mathrm{p}}\end{array}\right)=\left(\mathbf{A}^{t} \mathbf{A}\right)^{-1} \mathbf{A}^{t}(\mathrm{O}-\mathrm{C})$.

All necessary matrix inversion is made by the singular value decomposition.

Here $\delta \boldsymbol{u}^{0}=\left(\delta \boldsymbol{u}_{1}^{0}, . ., \delta \boldsymbol{u}_{\mathrm{n}}^{0}\right)^{t}$ are the corrections to the initial conditions of the $n$ asteroids with $\delta \boldsymbol{u}_{k}^{0}=\left(\delta \boldsymbol{r}^{0}, \delta \dot{\boldsymbol{r}}^{0}\right)$ standing for the corrections on the position and velocity of asteroid $k$ at the reference time $t_{0}$ with $\boldsymbol{r}=\boldsymbol{r}(t)=(x(t), y(t), z(t))$, $\dot{\boldsymbol{r}}=\dot{\boldsymbol{r}}(t)=(\dot{x}(t), \dot{y}(t), \dot{z}(t)), \boldsymbol{r}^{0}=\boldsymbol{r}\left(t_{0}\right)$. Then $\delta \boldsymbol{m}_{\mathrm{p}}=\left(\delta m_{1}, . ., \delta m_{\mathrm{p}}\right)^{t}$ are the corrections of the masses of the $p$ perturbers.

The matrix A contains the partial derivatives of the longitudes $\lambda$ of all minor planets involved in a close approach with respect to their initial parameters. $\mathbf{A}$ is block diagonal and one block has the following form,

$\mathbf{A}=\left(\begin{array}{ccccc}\mathbf{D}_{1} & 0 & \cdots & 0 & \mathbf{B}_{1} \\ 0 & \mathbf{D}_{2} & \ddots & \vdots & \mathbf{B}_{2} \\ \vdots & \ddots & \ddots & 0 & \vdots \\ 0 & \cdots & 0 & \mathbf{D}_{n} & \mathbf{B}_{n}\end{array}\right)$

where $\mathbf{D}_{k}$ is a $n_{\mathrm{obs}}^{k} \times 6$ matrix, $n_{\mathrm{obs}}^{k}$ being the number of observations for the asteroid $k$. The dimension of this matrix becomes $2 n_{\mathrm{obs}}^{k} \times 6$ if one takes into account the longitude and latitude together.

It represents the variations of the Gaia longitude $\lambda$ of the asteroid $\mathrm{k}$ with respect to its position and velocity in rectangular coordinates at the reference time $t_{0}$ for each observation. The elements of this matrix can be written as,

$\left[\mathbf{D}_{k}\right]_{i, j=1, . .6}=\left(\frac{\partial \lambda_{i}}{\partial x^{0}}, \frac{\partial \lambda_{i}}{\partial y^{0}}, \frac{\partial \lambda_{i}}{\partial z^{0}}, \frac{\partial \lambda_{i}}{\partial \dot{x}^{0}}, \frac{\partial \lambda_{i}}{\partial \dot{y}^{0}}, \frac{\partial \lambda_{i}}{\partial \dot{z}^{0}}\right)$

where $\lambda_{i}$ is the Gaia longitude of the asteroid $k$ for the $i$ th observation.
Table 3. Close approaches main statistics with a minimal distance $<0.5 \mathrm{AU}$, a deflection angle $>1$ mas and a perturber mass $>10^{-13} M_{\odot}$.

\begin{tabular}{lr}
\hline \hline Number of perturbing asteroids & 544 \\
Number of target asteroids & 12966 \\
Number of perturber-targets asteroids $^{1}$ & 436 \\
Number of close approaches & 28341 \\
\hline
\end{tabular}

${ }^{1}$ These asteroids belong to both categories of perturbing asteroids and target asteroids.

$\mathbf{B}_{k}$ represents the variations of the RGC longitude $\lambda$ of the asteroid $k$ with respect to the $p$ perturbing masses. $\mathbf{B}_{k}$ is a $n_{\mathrm{obs}}^{k} \times$ $p$ matrix.

$\left[\mathbf{B}_{k}\right]_{i, j=1, \ldots, p}=\left(\frac{\partial \lambda_{i}}{\partial m_{1}}, \cdots, \frac{\partial \lambda_{i}}{\partial m_{\mathrm{p}}}\right)$.

Add that the target asteroid has its own list of perturbers among all those considered and that consequently, $\partial \lambda_{i} / \partial m_{j}=0$ if the perturber $j$ does not perturb the target $k$ during the Gaia mission.

\subsection{The variational equations}

The evaluation of the partial derivatives requires their decomposition with respect to the rectangular coordinates. Thus, the elements of the matrix $\mathbf{A}$ can be written as,

$$
\begin{aligned}
& {\left[\mathbf{A}_{k}\right]_{i, j}=\sum_{q=1}^{3} \frac{\partial \lambda_{i}}{\partial r_{q}} \frac{\partial r_{q}}{\partial r_{j}^{0}}} \\
& {\left[\mathbf{B}_{k}\right]_{i, j}=\sum_{q=1}^{3} \frac{\partial \lambda_{i}}{\partial r_{q}} \frac{\partial r_{q}}{\partial m_{j}} .}
\end{aligned}
$$

The expression $\partial \lambda_{i} / \partial r_{q}$ is determined analytically while the part of the decomposition (5) expressing the variations of the rectangular coordinates $\boldsymbol{r}$ with respect to the free parameters are evaluated numerically. Thus, the variational Eqs. (7) and (8) describing their behavior (Kulikov 1950), are integrated together with the equation of motion (6).

$\ddot{\boldsymbol{r}}_{k}(t)=\boldsymbol{F}_{k}(t)$

where $\boldsymbol{F}_{k}$ is the force function which acts on the asteroid $k$.

The variational equations can be expressed by (Herget 1968),

$$
\begin{aligned}
\frac{\mathrm{d}^{2}}{\mathrm{~d} t^{2}}\left(\frac{\partial r_{q}}{\partial r_{j}^{0}}\right) & =\frac{\partial}{\partial r_{j}^{0}}\left(\ddot{r}_{q}\right) \\
& =\frac{\partial F_{q}}{\partial r_{j}^{0}} \\
& =\sum_{n=1}^{3}\left(\frac{\partial F_{q}}{\partial r_{n}} \frac{\partial r_{n}}{\partial r_{j}^{0}}+\frac{\partial F_{q}}{\partial \dot{r}_{n}} \frac{\partial \dot{r}_{n}}{\partial r_{j}^{0}}\right)
\end{aligned}
$$

$\frac{\mathrm{d}^{2}}{\mathrm{~d} t^{2}}\left(\frac{\partial r_{q}}{\partial m_{j}}\right)=\frac{\partial F_{q}}{\partial m_{j}}+\sum_{n=1}^{3}\left(\frac{\partial F_{q}}{\partial r_{n}} \frac{\partial r_{n}}{\partial m_{j}}+\frac{\partial F_{q}}{\partial \dot{r}_{n}} \frac{\partial \dot{r}_{n}}{\partial m_{j}}\right)$

The integration of the variational equations has been tested by direct comparison to a straight difference between two close orbits computed with two slight differences in the initial conditions and masses. Let $\zeta$ be one of these parameters to be fitted:

$\frac{\partial r_{q}}{\partial \zeta}=\frac{r_{q}(\zeta+\epsilon)-r_{q}(\zeta-\epsilon)}{2 \epsilon}$ 


\subsection{The dynamical model}

The theoretical positions and velocities of each asteroid have been computed for every observation using the Radau-Everhart integrator (Everhart 1985). The right-hand side function of the equation of motion (6) for an asteroid $\boldsymbol{F}_{k}$, includes the perturbations from the eight major planets, Pluto and the perturbing asteroids in the case of a target. Each target asteroid has its special list of perturbers determined previously by our close approaches selection procedure and the force model includes their perturbation throughout the entire span of the integration. The model is complete in the sense that it includes all selected targets and perturbers together. In addition, the solar quadrupole and the relativistic effects on the post-Newtonian approximation are also modelled. The initial epoch for the start of simulations is $t_{0}=$ JD 2456000.5 (January 2012) at which time the initial osculating elements of each asteroid are derived from the database of Bowell ${ }^{1}$. We have added a mass for each asteroid, based on existing data for the largest asteroids or determined from physical properties such as size and taxonomic type. For all assigned masses a density of $2.5 \mathrm{~g} / \mathrm{cm}^{3}$ has been adopted. It must be stressed that the detail of the mass distribution among the asteroids is limited, provided we have a database which reproduces something similar to the actual main belt. Our simulation and subsequent data processing remain consistent.

\subsection{The expected precision on the masses}

As noted earlier, Eq. (4) is solved for the initial conditions and the masses by least squares. If one has $N$ observed asteroids and $p$ perturbers, one has $6 N+p$ unknowns and typically $70 N$ observation equations. Note that $N \approx 10000-15000$ and $p \approx 500$. The solution for the corrections $\left(\delta \boldsymbol{u}^{0}, \delta \boldsymbol{m}_{\mathrm{p}}\right)^{t}$ is given by Eq. (4). From this system, it is possible to evaluate the formal precision $\sigma\left(\delta \boldsymbol{m}_{\mathrm{p}}\right)$ with which we will be able to determine the masses of perturbers $\boldsymbol{m}_{\mathrm{p}}$ knowing the precision of asteroids position.

If the normal matrix $\mathbf{A}^{t} \mathbf{A}$ is invertible, we obtain a relation between the variance-covariance matrix of the fitted parameters $\operatorname{cov}\left(\delta \boldsymbol{u}^{0}, \delta \boldsymbol{m}_{\mathrm{p}}\right)^{t}$ and the variance-covariance matrix $\operatorname{cov}(\mathrm{O}-\mathrm{C})$. More precisely one has,

$$
\begin{aligned}
\operatorname{cov}\left(\begin{array}{c}
\delta \boldsymbol{u}_{0} \\
\delta \boldsymbol{m}_{\mathrm{p}}
\end{array}\right) & =\operatorname{cov}\left[\left(\mathbf{A}^{t} \mathbf{A}\right)^{-1} \mathbf{A}^{t}(\mathrm{O}-\mathrm{C})\right] \\
& =\left(\mathbf{A}^{t} \mathbf{A}\right)^{-1} \mathbf{A}^{t}[\operatorname{cov}(\mathrm{O}-\mathrm{C})] \mathbf{A}\left(\mathbf{A}^{t} \mathbf{A}\right)^{-1} .
\end{aligned}
$$

The observation errors being different for various objects, we have weighed the observation equations. Each line of the matrix A corresponding to the $i$ th observation of the $k$ th asteroid has to be weighed according to the error on the position $\sigma_{k, i}$ (depending on the magnitude at the date of the observation $i$ ). By making the (very reasonable) assumption that the measures on the positions are independent and adding just a scale factor $\sigma_{0}$ to express the variance of the unit weight $\sigma_{0}^{2}$, the weighting matrix $\mathbf{W}$ is diagonal and can be written,

$\mathbf{W}=\left(\begin{array}{ccc}\ddots & & 0 \\ & \sigma_{0}^{2} / \sigma_{k, i}^{2} & \\ 0 & & \ddots\end{array}\right)$.

\footnotetext{
${ }^{1}$ URL: ftp://ftp. lowell.edu/pub/elgb/astorb.html
}

Equation (4) becomes

$$
\begin{aligned}
& \mathbf{W}^{1 / 2}(\mathrm{O}-\mathrm{C})=\mathbf{W}^{1 / 2} \mathbf{A}\left(\begin{array}{c}
\delta \boldsymbol{u}_{0} \\
\delta \boldsymbol{m}_{\mathrm{p}}
\end{array}\right) \\
& \Rightarrow\left(\begin{array}{c}
\delta \boldsymbol{u}_{0} \\
\delta \boldsymbol{m}_{\mathrm{p}}
\end{array}\right)=\left(\mathbf{A}^{t} \mathbf{W A}\right)^{-1} \mathbf{A}^{t} \mathbf{W}(\mathrm{O}-\mathrm{C}) .
\end{aligned}
$$

Consequently, we obtain the following relations,

$\operatorname{cov}[\mathbf{W}(\mathrm{O}-\mathrm{C})]=\sigma_{0}^{2} I$

$\operatorname{cov}\left(\begin{array}{l}\delta \boldsymbol{u}_{0} \\ \delta \boldsymbol{m}_{\mathrm{p}}\end{array}\right)=\sigma_{0}^{2}\left(\mathbf{A}^{t} \mathbf{W} \mathbf{A}\right)^{-1}$

To evaluate the precision of the masses of the perturbers is equivalent to computing particular elements of the matrix $\left(\mathbf{A}^{t} \mathbf{W A}\right)^{-1}$, which will be represented by the $p \times p$ matrix $\mathbf{U}$. So, the covariance of the parameters $\delta \boldsymbol{m}_{\mathrm{p}}$ and consequently, their variance can be expressed

$\operatorname{cov}\left(\delta \boldsymbol{m}_{\mathrm{p}}\right)=\sigma_{0}^{2} \mathbf{U}^{-1}$
$\sigma\left(\delta \boldsymbol{m}_{\mathrm{p}}\right)_{i}=\sqrt{\operatorname{cov}\left(\delta \boldsymbol{m}_{\mathrm{p}}\right)_{i, i}} \quad i=1, \ldots, p$.

From the different matrices $\left(\mathbf{D}_{k}, \mathbf{B}_{k}\right)_{k=1, \ldots, n}$ which make up the global partial derivatives matrix $\mathbf{W}^{1 / 2} \mathbf{A}$, we can evaluate the components of the matrix $\mathbf{U}$ by the following formula (Söderhjelm et al. 1982)

$$
\mathbf{U}=\sum_{k=1}^{N}\left(\mathbf{B}_{k}^{t} \mathbf{B}_{k}-\mathbf{B}_{k}^{t} \mathbf{D}_{k}\left(\mathbf{D}_{k}^{t} \mathbf{D}_{k}\right)^{-1} \mathbf{D}_{k}^{t} \mathbf{B}_{k}\right)
$$

where $N$ is the total number of treated asteroids.

As said earlier, the inversion of the matrix $\mathbf{U}$ is made by the singular value decomposition method. So, the $p \times p$ matrix $\mathbf{U}$ can be written as the product of,

\section{$\mathbf{U}=\mathbf{L W} \mathbf{V}^{t}$}

where $\mathbf{L}$ is an $p \times p$ column-orthogonal matrix, $\mathbf{W}$ an $p \times p$ diagonal matrix with the singular values, $\mathbf{V}$ an $p \times p$ orthogonal matrix and $p$ the total number of perturbers. Consequently, the inverse matrix $\mathbf{U}^{-1}$ is equal to,

$\mathbf{U}^{-1}=\mathbf{V} \mathbf{W}^{-1} \mathbf{L}^{t}$

\section{Results}

All the above computations have been implemented with a realistic simulation of the Gaia observations: the date of each observation was computed by taking into account the position of Gaia space probe on its Lissajous orbit, the complex Gaia scanning law (Sect. 2.2) and the motion of the asteroids, including the propagation of light between the asteroids and Gaia. The apparent magnitude and the phases are then computed at the transit time to compute the observation accuracy. Each line of the observation matrix is then determined from the numerical integration of the equation of motions and of the variational equations.

The main result is shown in Table 4 with the statistical distribution of the precision on the masses. There are about 42 asteroids for which the mass can be ascertained to better than $10 \%$ and this number rises to 150 within a $50 \%$ precision. The first two, 1 Ceres and 4 Vesta are determined with a formal precision of $0.07 \%$ and there are an additional 5 within $1 \%$. Table 7 lists the individual objects having at the end a mass recovered with a relative precision smaller than $10 \%$. We can see the number of targets for each mass determination, the number of close approaches which will be out of the Gaia observations range, the 
Table 4. Number of masses determined in each class of the relative precision.

\begin{tabular}{cc}
\hline \hline \multicolumn{2}{c}{ Number of perturbers } \\
\hline Total & 544 \\
$\sigma(m) / m<0.1 \%$ & 2 \\
$\sigma(m) / m<1 \%$ & 7 \\
$\sigma(m) / m<10 \%$ & 42 \\
$\sigma(m) / m<15 \%$ & 56 \\
$\sigma(m) / m<20 \%$ & 76 \\
$\sigma(m) / m<30 \%$ & 113 \\
$\sigma(m) / m<40 \%$ & 135 \\
$\sigma(m) / m<50 \%$ & 150 \\
\hline
\end{tabular}

Table 5. Distribution of the amplitude $c_{i j}$ with respect to the associated period $P$ for asteroids having an influence on the Mars ephemerides. The period $P$ is expressed in years.

\begin{tabular}{|c|c|c|c|c|}
\hline \multicolumn{2}{|c|}{ Asteroid } & \multicolumn{3}{|c|}{$\Delta a_{\text {mars }}$} \\
\hline \multirow[t]{2}{*}{$n^{\circ}$} & \multirow[t]{2}{*}{ IAU } & \multicolumn{3}{|c|}{ Number of amplitudes $c_{i j}^{1}>\times 10^{-10} \mathrm{AU}$} \\
\hline & & $P<100$ & $100 \leq P<200$ & $200 \leq P<500$ \\
\hline 1 & Ceres & 8 & 0 & 0 \\
\hline 4 & Vesta & 5 & 0 & 0 \\
\hline 2 & Pallas & 1 & 0 & 0 \\
\hline 7 & Iris & 2 & 0 & 0 \\
\hline 324 & Bamberga & 0 & 1 & 0 \\
\hline 3 & Juno & 0 & 1 & 0 \\
\hline 6 & Hebe & 0 & 0 & 2 \\
\hline 511 & Davida & 0 & 0 & 1 \\
\hline 20 & Massalia & 0 & 0 & 1 \\
\hline 344 & Desiderata & 0 & 0 & 0 \\
\hline \multicolumn{2}{|c|}{ Asteroid } & \multicolumn{3}{|c|}{$\overline{\Delta \lambda_{\text {mars }}}$} \\
\hline \multirow[t]{2}{*}{$n^{\circ}$} & \multirow[t]{2}{*}{ IAU } & \multicolumn{3}{|c|}{ Number of amplitudes $\quad c_{i j}^{1}>\times 10^{-8}$ radians } \\
\hline & & $P<100$ & $100 \leq P<200$ & $200 \leq P<500$ \\
\hline 1 & Ceres & 1 & 0 & 0 \\
\hline 4 & Vesta & 1 & 0 & 0 \\
\hline 2 & Pallas & 1 & 0 & 0 \\
\hline 7 & Iris & 0 & 0 & 0 \\
\hline 324 & Bamberga & 0 & 1 & 0 \\
\hline 3 & Juno & 0 & 0 & 0 \\
\hline 6 & Hebe & 0 & 0 & 2 \\
\hline 511 & Davida & 0 & 0 & 2 \\
\hline 20 & Massalia & 0 & 0 & 1 \\
\hline 344 & Desiderata & 0 & 0 & 1 \\
\hline
\end{tabular}

formal precision and, the reference masses used in this work and finally, the relative precision in percent.

It is interesting to point out that an accurate mass of 2 Pallas can be derived with Gaia, whereas, because of its large inclination (Michalak 2000) there are rare encounters with other bright asteroids. Becoming more faint allows observation of significant close approaches between Pallas and faint asteroids which have not been observed over a sufficient period of time from the Earth. There is for example a very close approach $(\approx 0.006 \mathrm{AU})$ in 2014 with 17278 Viggh yielding a deflection of 40 mas, very significant for Gaia astrometry, but not for standard ground-based monitoring. With a mass probably comparable to that of Vesta, but poorly known, this is one of the most perturbing asteroid for solar system objects. To a lesser degree, having a more precise measure of its mass will also allow a reduction, when Pallas becomes a target, in the error on the mass determination of certain perturbers due to the influence of correlation explained in Sect. 2.1.
Table 6. Distribution of the asteroids with respect to their taxonomic class.

\begin{tabular}{ccc}
\hline \hline $\begin{array}{c}\text { Taxonomic } \\
\text { Class } \\
\text { (SMASSII) }\end{array}$ & $\begin{array}{c}\text { Number of asteroids } \\
\text { from Table } 1\end{array}$ & $\begin{array}{c}\text { Number of asteroids } \\
\text { from Table 7 } \\
\sigma(m) / m<10 \%\end{array}$ \\
\hline $\mathrm{S}$ & 2 & 7 \\
$\mathrm{~S}_{\mathrm{k}}$ & 1 & 2 \\
$\mathrm{C}$ & 5 & 9 \\
$\mathrm{C}_{\mathrm{b}}$ & 1 & 1 \\
$\mathrm{C}_{\mathrm{h}}$ & 1 & 4 \\
$\mathrm{~B}$ & 1 & 6 \\
$\mathrm{~V}$ & 1 & 1 \\
$\mathrm{X}$ & 2 & 1 \\
$\mathrm{X}_{\mathrm{c}}$ & 0 & 1 \\
$\mathrm{X}_{\mathrm{e}}$ & 0 & 1 \\
$\mathrm{~K}$ & 0 & 1 \\
$\mathrm{~L}$ & 0 & 1 \\
\hline
\end{tabular}

As mentioned in Sect. 1, the precision of the Mars ephemerides at the present time, is limited by the uncertainty of the masses of the biggest asteroids of the main belt. We have started an analytical investigation in order to give a preliminary list of these asteroids which have the greatest influence on the Mars orbit and to see how this can be improved with Gaia. This analysis is based on a software developed by Simon $(1986,1987)$, which permits expression of the perturbations $\Delta \sigma_{k}$ of a asteroid on the six Mars equinoctial elements $\sigma_{k=1, . .6}=(a, \lambda, k, h, q, p)$ during 1000 years by using harmonic decomposition with respect to the mean mean longitude of Mars $\bar{\lambda}$ and the mean mean longitude of the asteroid $\bar{\lambda}^{\prime}, a$ being the semi major axis, $\lambda=\varpi+v$ with $\varpi$ the longitude of perihelion and $v$ the true anomaly, $k=e \cos \varpi, h=e \sin \varpi, q=\sin (i / 2) \cos \Omega$, $p=\sin (i / 2) \sin \Omega$, and $\Omega$ the longitude of the ascending node, $e$ the eccentricity and $i$ the inclination.

$\Delta \sigma_{k}$ can be written by the formula,

$\Delta \sigma_{k}\left(\bar{\lambda}, \bar{\lambda}^{\prime}\right)=\sigma_{k}^{\mathrm{sec}} t+\sum_{i, j} a_{i j}^{k} \cos \left(i \bar{\lambda}+j \bar{\lambda}^{\prime}\right)+b_{i j}^{k} \sin \left(i \bar{\lambda}+j \bar{\lambda}^{\prime}\right)$.

We have applied this decomposition for each perturbation of the 300 most massive asteroids, and have focused on those which act upon the semi major axis and longitude of Mars. We have the asteroids by selecting first the two amplitudes $c_{i j}^{k}=\left[\left(a_{i j}^{k}\right)^{2}+\left(b_{i j}^{k}\right)^{2}\right]^{1 / 2}(k=1,2)$ greater than $1 . \times 10^{-10} \mathrm{AU}$ concerning the semi major axis of $\operatorname{Mars}(k=1)$, and $1 . \times 10^{-8}$ radians for its longitude $(k=2)$. This threshold value was chosen according to the amplitude obtained for Ceres, a major perturber of Mars. The second filter consisted excluding the associated pe$\operatorname{riod} P_{i j}^{k}$ superior to 500 years, which is defined by,

$$
P_{i j}^{k}=\frac{2 \pi}{i \times n_{\text {mars }}+j \times n^{\prime}}
$$

with $i \in\{0,1, \ldots, 2 p-1\}$ and $j \in\left\{0,1, \ldots, 2 p^{\prime}-1\right\}$ where $n_{\text {mars }}$ and $n^{\prime}$ are respectively the mean motion of Mars and the considered asteroid, and $p$ and $p^{\prime}$ two fixed integers. In Table 5, we have indicated for each selected asteroid, the number of coefficients obtained in the expression of their perturbation on the semi-major axis $\Delta a_{\text {mars }}$ and the longitude $\Delta \lambda_{\text {mars }}$ of Mars, as well as the interval in which the corresponding period is located. The major role played by the big threes, Ceres, Pallas and Vesta appears clearly in this table. But the seven others were less expected and only one (344 Desiderata) does not belong to our list 
Table 7. The formal precisions on the perturbers masses. The binary asteroid are marked by suffix $b$.

\begin{tabular}{|c|c|c|c|c|c|c|c|}
\hline \multicolumn{2}{|c|}{ Asteroid } & \multirow{2}{*}{$\begin{array}{c}\text { Number of } \\
\text { target asteroids }\end{array}$} & \multirow{2}{*}{$\begin{array}{c}\text { Number of } \\
\text { close approaches } \\
\text { not observed }\end{array}$} & \multirow{2}{*}{$\begin{array}{c}\text { Sigma } \\
M_{\odot}\end{array}$} & \multirow{2}{*}{$\begin{array}{c}\text { Mass } \\
M_{\odot}\end{array}$} & \multirow[t]{2}{*}{ Sigma/mass } & \multirow{2}{*}{$\begin{array}{c}\text { Taxonomic class } \\
\text { SMASSII }\end{array}$} \\
\hline $\mathrm{N}^{\circ} \mathrm{IAU}$ & Name & & & & & & \\
\hline 4 & Vesta & 2104 & 646 & $9.29 \times 10^{-14}$ & $1.30 \times 10^{-10}$ & $0.071 \%$ & V \\
\hline 1 & Ceres & 1663 & 469 & $3.50 \times 10^{-13}$ & $4.50 \times 10^{-10}$ & $0.078 \%$ & $\mathrm{C}$ \\
\hline 14 & Irene & 1030 & 131 & $1.03 \times 10^{-13}$ & $2.60 \times 10^{-11}$ & $0.397 \%$ & S \\
\hline 27 & Euterpe & 812 & 181 & $5.14 \times 10^{-14}$ & $1.00 \times 10^{-11}$ & $0.514 \%$ & S \\
\hline 10 & Hygiea & 1026 & 201 & $3.25 \times 10^{-13}$ & $4.70 \times 10^{-11}$ & $0.691 \%$ & $\mathrm{C}$ \\
\hline 52 & Europa & 859 & 320 & $2.19 \times 10^{-13}$ & $2.40 \times 10^{-11}$ & $0.913 \%$ & $\mathrm{C}$ \\
\hline 2 & Pallas & 466 & 13 & $1.28 \times 10^{-12}$ & $1.30 \times 10^{-10}$ & $0.983 \%$ & B \\
\hline 15 & Eunomia & 527 & 159 & $2.79 \times 10^{-13}$ & $1.50 \times 10^{-11}$ & $1.860 \%$ & S \\
\hline 46 & Hestia & 136 & 25 & $2.25 \times 10^{-14}$ & $1.20 \times 10^{-12}$ & $1.880 \%$ & $\mathrm{X}_{\mathrm{c}}$ \\
\hline 511 & Davida & 430 & 86 & $6.17 \times 10^{-13}$ & $3.00 \times 10^{-11}$ & $2.060 \%$ & $\mathrm{C}$ \\
\hline 88 & Thisbe & 341 & 74 & $1.06 \times 10^{-13}$ & $5.00 \times 10^{-12}$ & $2.120 \%$ & B \\
\hline 19 & Fortuna & 911 & 313 & $1.86 \times 10^{-13}$ & $8.30 \times 10^{-12}$ & $2.240 \%$ & $\mathrm{C}_{\mathrm{h}}$ \\
\hline 16 & Psyche & 1051 & 343 & $3.42 \times 10^{-13}$ & $1.40 \times 10^{-11}$ & $2.440 \%$ & $X$ \\
\hline 29 & Amphitrite & 608 & 132 & $1.68 \times 10^{-13}$ & $6.00 \times 10^{-12}$ & $2.800 \%$ & S \\
\hline 20 & Massalia & 188 & 58 & $6.33 \times 10^{-14}$ & $1.90 \times 10^{-12}$ & $3.330 \%$ & S \\
\hline 24 & Themis & 678 & 145 & $4.07 \times 10^{-13}$ & $1.20 \times 10^{-11}$ & $3.390 \%$ & B \\
\hline 64 & Angelina & 405 & 151 & $1.37 \times 10^{-13}$ & $4.00 \times 10^{-12}$ & $3.430 \%$ & $X_{e}$ \\
\hline 7 & Iris & 229 & 47 & $1.77 \times 10^{-13}$ & $5.00 \times 10^{-12}$ & $3.540 \%$ & $\mathrm{~S}$ \\
\hline 704 & Interamnia & 404 & 24 & $1.06 \times 10^{-12}$ & $3.00 \times 10^{-11}$ & $3.550 \%$ & B \\
\hline 241 & Germania & 163 & 21 & $1.26 \times 10^{-13}$ & $3.00 \times 10^{-12}$ & $4.210 \%$ & B \\
\hline 324 & Bamberga & 127 & 38 & $4.70 \times 10^{-13}$ & $1.00 \times 10^{-11}$ & $4.700 \%$ & / \\
\hline 45 & Eugenia & 544 & 140 & $3.00 \times 10^{-13}$ & $6.20 \times 10^{-12}$ & $4.830 \%$ & $\mathrm{C}$ \\
\hline 94 & Aurora & 273 & 74 & $3.70 \times 10^{-13}$ & $7.50 \times 10^{-12}$ & $4.930 \%$ & $\mathrm{C}$ \\
\hline 203 & Pompeja & 140 & 36 & $4.88 \times 10^{-14}$ & $9.80 \times 10^{-13}$ & $4.980 \%$ & / \\
\hline 150 & Nuwa & 260 & 100 & $1.15 \times 10^{-13}$ & $2.20 \times 10^{-12}$ & $5.230 \%$ & $\mathrm{C}_{\mathrm{b}}$ \\
\hline 51 & Nemausa & 64 & 15 & $1.09 \times 10^{-13}$ & $2.00 \times 10^{-12}$ & $5.430 \%$ & $\mathrm{C}_{\mathrm{h}}$ \\
\hline 48 & Doris & 436 & 104 & $3.83 \times 10^{-13}$ & $6.80 \times 10^{-12}$ & $5.630 \%$ & $\mathrm{C}_{\mathrm{h}}$ \\
\hline 423 & Diotima & 164 & 33 & $3.22 \times 10^{-13}$ & $5.70 \times 10^{-12}$ & $5.650 \%$ & $\mathrm{C}$ \\
\hline 128 & Nemesis & 375 & 58 & $2.51 \times 10^{-13}$ & $4.20 \times 10^{-12}$ & $5.970 \%$ & $\mathrm{C}$ \\
\hline 532 & Herculina & 109 & 31 & $5.49 \times 10^{-13}$ & $9.00 \times 10^{-12}$ & $6.100 \%$ & I \\
\hline 12 & Victoria & 19 & 7 & $5.53 \times 10^{-14}$ & $9.00 \times 10^{-13}$ & $6.150 \%$ & $\mathrm{~L}$ \\
\hline 11 & Parthenope & 282 & 74 & $1.45 \times 10^{-13}$ & $2.30 \times 10^{-12}$ & $6.300 \%$ & $\mathrm{~S}_{\mathrm{k}}$ \\
\hline 89 & Julia & 17 & 6 & $1.41 \times 10^{-13}$ & $2.20 \times 10^{-12}$ & $6.420 \%$ & $\mathrm{~K}$ \\
\hline 6 & Hebe & 120 & 33 & $4.51 \times 10^{-13}$ & $7.00 \times 10^{-12}$ & $6.440 \%$ & $S$ \\
\hline 451 & Patientia & 108 & 28 & $4.73 \times 10^{-13}$ & $7.10 \times 10^{-12}$ & $6.660 \%$ & l \\
\hline 120 & Lachesis & 150 & 71 & $2.34 \times 10^{-13}$ & $3.30 \times 10^{-12}$ & $7.090 \%$ & $\mathrm{C}$ \\
\hline 3 & Juno & 133 & 33 & $5.77 \times 10^{-13}$ & $8.00 \times 10^{-12}$ & $7.210 \%$ & $\mathrm{~S}_{\mathrm{k}}$ \\
\hline 554 & Peraga & 40 & 5 & $4.05 \times 10^{-14}$ & $5.50 \times 10^{-13}$ & $7.360 \%$ & l \\
\hline 8 & Flora & 168 & 36 & $1.68 \times 10^{-13}$ & $2.20 \times 10^{-12}$ & $7.620 \%$ & 1 \\
\hline 59 & Elpis & 153 & 56 & $2.36 \times 10^{-13}$ & $2.80 \times 10^{-12}$ & $8.420 \%$ & B \\
\hline 9 & Metis & 246 & 76 & $2.42 \times 10^{-13}$ & $2.70 \times 10^{-12}$ & $8.970 \%$ & 1 \\
\hline 41 & Daphne & 17 & 2 & $3.28 \times 10^{-13}$ & $3.30 \times 10^{-12}$ & $9.950 \%$ & $\mathrm{C}_{\mathrm{h}}$ \\
\hline
\end{tabular}

of asteroids in Table 7 for which we expect a precision on the mass lower than $10 \%$. However it may show up since the number of targets in the actual mission will be $\sim 350000$ rather than the $\sim 20000$ used in this exercise.

The Gaia mission will also play an important role in the knowledge of the physical properties of asteroids. In Table 6 we compare the distribution of the asteroids from Tables 1 and 7 with respect to their taxonomic classes from SMASSII data (Bus $\&$ Binzel 2002), which allows us to catch a glimpse of the importance that Gaia will have. The study of a possible relationship between the asteroids bulk density and their taxonomic classes will become truly possible. We note that the number of densities we could determine with Gaia is distributed on a larger set of taxonomic classes (12 instead of 8 ) and that their number for each class is often much larger. More precisely, 4 groupings, among them two of three major ones S, C-type asteroids, as well as the $B$ and $C_{h}$ classes will have a set of densities much larger than at present.
Futhermore, the computed densities with the expected masses from Gaia will very often be much more precise than those made at the present time. Taking the example of the asteroid 704 Interamnia for which we have a density bearing large error bars partly due to the mass uncertainty. Its IRAS diameter is $D=(316.6 \pm 5.2) \mathrm{km}$, and in consequence, we find a volume $V=(16.6 \pm 0.8) \times 10^{15} \mathrm{~m}^{3}$ for a mass estimated to $(3.5 \pm$ $0.9) \times 10^{-11} M_{\odot}\left(\right.$ Michalak 2001). Thus, its bulk density is $\rho_{\mathrm{b}}=$ $(4.21 \pm 1.29) \times 10^{3} \mathrm{~kg} / \mathrm{m}^{3}$, the density error being estimated by the formula (Birlan 2000),

$\Delta \rho_{\mathrm{b}}=\frac{\Delta M}{V}+\frac{M \Delta V}{V^{2}}$ with $\rho_{\mathrm{b}}=\frac{M}{V}$.

Considering a mass of 704 interamnia similar to that found by Michalak but determined with the relative precision that Gaia will permit, so $3.55 \%$, the error on the density would become $\Delta \rho_{\mathrm{b}}=0.36 \times 10^{3} \mathrm{~kg} / \mathrm{m}^{3}$, almost 4 times less than the current error. Nevertheless, we are aware of the preponderance of 
the uncertainty of the asteroid diameter in the density estimation depending on the inverse of the cube of diameter. In consequence, the knowledge of the asteroid masses from Gaia will be increased in scale when it will be associated with future missions or ground-based observations devoted to the imagery of the asteroids shape. So, the mass associated with the volume, which will be much better determined, will allow a good approximation of its porosity to be obtained, a precious clue for the investigation of its collisional history.

\section{Conclusions}

1. From a subset of target asteroids taken among the first 20000 that Gaia will observe, we found that 42 masses could be derived with a precision better than $10 \%$ and 150 with a precision better than $50 \%$. Keeping in mind that Gaia will actually observe about 350000 asteroids, so that the number of close approaches is potentially much larger, one can expect more mass determinations at this precision level.

2. We have designed and implemented a relevant mathematical and statistical approach to solve globally the determination of the masses of $\sim 200$ perturbers together with the orbital parameters of a very large number of targets.

3. A possible contribution of ground-based observations is being studied to define in which scope they would be interesting for the mass determination with Gaia. Another study is being initiated to estimate the impact of the mass determination with the Gaia mission on the further improvement of the Mars ephemeris.

\section{References}

Aslan, Z., Gumerov, R. I., Hudkova, L. A., et al. 2006, 26th meeting of the IAU, Joint Discussion 16, 67, 16

Bange, J. 1998, A\&A, 340, L1

Behrend, R., Bernasconi, L., Roy, R., et al. 2006, A\&A, 446, 1177

Belton, M. J. S., Veverka, J., Thomas, P. C., et al. 1995, Nature, 374, 783

Birlan, M. 2000, Earth Moon and Planets, 88, 1

Bowell, E. 2001, Asteroid Orbital Elements Database, http://www. lowell.edu/users/elgb/

Britt, D. T., Yeomans, D., Housen, K., \& Consolmagno, G. 2002, Asteroids III, ed. W. F. Bottke, A. Cellino, P. Paolicchi, \& R. P. Binzel (Tucson: University of Arizona Press), 485

Bus, J. S., \& Binzel, R. P. 2002, Icarus, 158, 146

Carpino, M., \& Knežević, Z. 1996, in Dynamics, Ephemerides, and Astrometry of the Solar System, ed. S. Ferraz-Mello et al. (Dordrecht: Kluwer), Proc. IAU Symp., 172, 203
Chesley, S. R., Owen, Jr., W. M., Hayne, E. W., et al. 2005, BAAS, 37, 524

Everhart, E. 1985, in proceedings of IAU Colloq. 83, ed. A. Carusi, \& G. B. Valsecchi (Astrophysics and Space Science Library), 115, 185

Goffin, E. 1991, A\&A, 249, 563

Goffin, E. 2001, A\&A, 365, 627

Herget, P. 1968, AJ, 73, 737

Hertz, H. G. 1966, IAU Circ., 1983

Hilton, J. L. 1999, AJ, 117, 1077

Hilton, J. L. 2002, Asteroids III, ed. W. F. Bottke, A. Cellino, P. Paolicchi, \& R. P. Binzel (Tucson: University of Arizona Press), 103

Kochetova, O. M. 2004, Sol. Syst. Res., 38, 66

Kovačević, A., \& Kuzmanoski, M. 2005, Serb. Astron. J., 171, 37

Kulikov, D. K. 1950, Bull. Inst. Theoret. Astron., 4, 311

Kuzmanoski, M. 1996, in Dynamics, Ephemerides, and Astrometry of the Solar System, ed. S. Ferraz-Mello et al. (Dordrecht: Kluwer), Proc. IAU Symp., 72,207

Kuzmanoski, M., \& Kovačević, A. 2002, A\&A, 395, L17

Kuznetsov, V. B. 2001, Soobshch. IPA RAN, 138

Marchis, F., Descamps, P., Hestroffer, D., et al. 2003, Icarus, 165, 112

Marchis, F., Hestroffer, D., Descamps, P., et al. 2005, Icarus, 178, 450

Marchis, F., Descamps, P., Hestroffer, D., \& Berthier, J. 2005, Nature, 436, 822

Marchis, F., Hestroffer, D., Descamps, P., et al. 2006, Nature, 439, 565

Margot, J.-L., Brown, M. E., de Pater, I., \& Roe, H. 2001, in Asteroids 2001, From Piazzi to the Third Millenium, 19

Merline, W. J., Close, L. M., Dumas, C., et al. 1999, Nature, 401, 565

Merline, W. J., Weidenschilling, S. J., Durda, D. D., et al. 2002, Asteroids III, ed. W. F. Bottke, A. Cellino, P. Paolicchi, \& R. P. Binzel (Tucson: University of Arizona Press), 289

Michalak, G. 2000, A\&A, 360, 363

Michalak, G. 2001, A\&A, 374, 703

Mignard, F. 2005, in proceedings of the symposium ESA 576, ed. C. Turon, K. S. O'Flaherty, \& M. A. C. Perryman (ESA Publications Division), 5

Miller, J. K., Konopliv, A. S., Antreasian, P. G., et al. 2002, Icarus, 155, 3

Petit, J. M., Durda, D. D., Greenberg, R., Hurford, T. A., \& Geissler, P. E. 1997, Icarus, 130, 177

Pitjeva, E. V. 2001, A\&A, 371, 760

Simon, J.-L. 1986, Note. Sci. Techn. Bureau Long., S013

Simon, J.-L. 1987, A\&A, 175, 303

Sitarski, G., \& Todorovic-Juchniewicz, B. 1992, Acta Astron., 42, 139

Sitarski, G., \& Todorovic-Juchniewicz, B. 1995, Acta Astron., 45, 673

Söderhjelm, S., \& Lindegren, L. 1982, A\&A, 110, 156

Standish, E. M. Jr., \& Fienga, A. 2002, A\&A, 384, 322

Viateau, B., \& Rapaport, M. 1995, A\&AS, 111, 305

Viateau, B., \& Rapaport, M. 1997, in Proceedings of the ESA Symposium Hipparcos-Venice'97, ed. B. Battrick, ESA SP-402, 91

Viateau, B., \& Rapaport, M. 1998, A\&A, 334, 729

Viateau, B., \& Rapaport, M. 2001, A\&A, 370, 602

Vitagliano, A., \& Stoss, R. M. 2006, A\&A, 455, L29

Van Der Waerden, B. L. 1969, Mathematical Statistics (Berlin: Springer)

Williams, G. V. 1992, in Asteroids, Comets, and Meteors 1991, ed. A. W. Harris, $\&$ E. Bowell (Houston: Lunar and Planetary Institute), 641

Yeomans, D. K., \& 12 colleagues 1998, Science, 278, 2106

Zhang, N. F. 2006, Metrologia, 43, 195

Zappalà, V., \& Cellino, A. 2002, GAIA: a european space project, ed. O. Bienaymé, \& C. Turon (EAS Publications Series), 2, 343 